\title{
In silico comparative molecular docking analysis and analysis of the anti-inflammatory mechanisms of action of tanshinone from Salvia miltiorrhiza
}

\author{
ZHENGSHANG RUAN $^{1 *}$, LEI NIU ${ }^{2 *}$, LI HAN $^{3 *}$, RONGRONG REN ${ }^{1}$, \\ ZHENYU XU ${ }^{4}$, WENWEN DONG ${ }^{1}$ and LAI JIANG ${ }^{1}$

\begin{abstract}
Departments of ${ }^{1}$ Anesthesiology and Surgical Intensive Care Unit, ${ }^{2}$ Emergency and
${ }^{3}$ Geriatrics, Xinhua Hospital, Shanghai Jiaotong University School of Medicine, Shanghai 200092;

${ }^{4}$ Department of Emergency, Shanghai Seventh People's Hospital, Shanghai 200437, P.R. China
\end{abstract}

Received May 14, 2019; Accepted June 17, 2019

DOI: $10.3892 /$ etm.2019.7679

\begin{abstract}
Tanshinones are a class of abietane diterpene compounds extracted from Salvia miltiorrhiza, and have been used for medical purposes in traditional Chinese medicinal practices. This herb has been used in the treatment of chronic obstructive pulmonary disease (COPD), breast cancer and inflammatory disorders. This study examined the anti-inflammatory properties of tanshinones. In addition, lipid-soluble compounds which were specific to Tanshinone class were also highlighted, out of which two compounds, dihydrotanshinone I and cryptotanshinone were selected with the aim of creating a new research perspective in order to further elucidate the mechanisms of the pathogenesis of inflammatory diseases. Moreover, interaction analyses were carried out successfully to determine the interactions formed between both dihdrotanshinone I and cryptotanshinone, and target proteins. The bioactivity properties and various other pharmacokinetic and pharmacodynamic analyses discerned that crytptotanshinone was more effective dihydrotanshinone and more 'drug-like' than its counterpart. It was found to have a better solubility and permeability, and thus has potential for use as an anti-inflammatory agent.
\end{abstract}

\section{Introduction}

Tanshinones are a class of abietane diterpene compounds extracted from the well-known Chinese herbal plant, Salvia

Correspondence to: Professor Lai Jiang or Dr Wenwen Dong, Department of Anesthesiology and Surgical Intensive Care Unit, Xinhua Hospital, Shanghai Jiaotong University School of Medicine, 1665 Kongjiang Road, Yangpu, Shanghai 200092, P.R. China

E-mail: jianglai@xinhuamed.com.cn

E-mail: dongwenwen@xinhuamed.com.cn

${ }^{*}$ Contributed equally

Key words: tanshinone, anti-inflammatory, molecular docking, anti-cancer, Chinese herbal plant miltiorrhiza (Red sage or Chinese sage or Danshen) viz., a perennial plant with a height between 90 to 1,200 m, and which is highly valued for its roots which are religiously employed for medical purposes in traditional Chinese medicinal practices (1). The plant is a 'part and parcel' of the Chinese and the Japanese territories and usually grows in grassy, yet mushy locations, such as forests or hillsides (2). This herb has been already tested for the treatment of chronic obstructive pulmonary disease (COPD), and has shown to be highly effective $(3,4)$. Moreover, the abietane-type diterpene compound, tanshinone, has been used as a therapeutic remedy for a myriad of diseases, such as coronary artery and vascular diseases, stroke, hyperlipidemia, endangiitis, arthritis and hepatitis (5). The compound has also been employed for the treatment of breast cancer and inflammatory disorders (1). This study examined the anti-inflammatory properties of this compound.

Inflammation usually occurs when the body comes into contact with any harmful pathogen, irritant or when cell damage occurs. This type of inflammation is simply a function of self-defense, or it can occur as a protective mechanism of the body (6). With the help of an exhaustive literature recapitulation, it can be discerned that there are $>100$ compounds which have been screened from Salvia miltiorrhiza and have been classified as per their chemical properties and structural capabilities. There are basically two categories in which the classification has been laid down: i) Water-soluble compounds, which comprise compounds, such as salvianolic A-G and lithospermic acid B; and ii) lipid-soluble category compounds which encapsulate the compounds tanshinone IIA, dihydrotanshinone I (https://pubchem.ncbi.nlm.nih.gov/compound/11425923; https://toxnet.nlm.nih.gov/cgi-bin/sis/search2/r?dbs+hsdb:@ term+@rn+@rel+87205-99-0) and cryptotanshinones (https://pubchem.ncbi.nlm.nih.gov/compound/160254) $(7,8)$. The lipid-soluble categories of the compounds have been shown to have anti-inflammatory properties (5).

In this study, we focused our attention on tanshinone compounds which have the potential to act as anti-inflammatory agents for various inflammation-based diseases. Furthermore, we targeted these compounds for a molecular docking and interaction analyses to their counterpart proteins. 
The basic mode of operation of this study has been showcased in the form of a flowchart as shown in Fig. 1.

\section{Materials and methods}

Compounds. Query tanshinone compounds were retrieved from the ChEMBL database (9) along with their target proteins. A list of the query compounds and their target proteins is presented in Tables I-V. An extensive literature recapitulation was accomplished in order to search for lipid-soluble compounds, and in particular for tanshinone compounds. Initially, 18 compounds were retrieved which exhibited anti-inflammatory acitivity (5). An exhaustive filtering was carried out so as to retrieve the best, optimal and specific tanshinone compounds. By using the ChEMBL database, 4 best keyword matching the compounds were obtained namely, tanshinone IIA (CHEMBL187266), tanshinone IIA sulfone (CHEMBL1473252), dihydrotanshinone I (CHEMBL227075) and crytotanshinone (CHEMBL187460). Out of these 4, only 2, dihydrotanshinone I and cryptotanshinone were selected for further analysis as they had a greater number of similar target proteins.

Determination of pharmacokinetic and pharmacodynamic properties and bioactivity of tanshinone compounds. The acknowledgement of the binding of tanshinone compounds to their target proteins was accomplished using Molinspiration (http://www.molinspiration.com/cgi-bin/properties) and DrugBank $(10,11)$. ADME expands to Absorption, Distribution, Metabolism and Excretion. ADME properties plays pivotal role during the process of drug design due to the fact that these account for the failure of approximately $60 \%$ of all drugs during clinical phases. Traditionally, these properties are assessed at the end of the drug development process, but now-a-days it is applied at an early phase in order to filter out molecules with poor ADME properties. DrugBank $(10,11)$ is an inclusive, open and freely-available online database which encases information on drugs and their targets. It a type of encyclopedia of drugs. Molinspiration is a cheminformatics tool, which is an online based tool used to calculate molecular properties, such as molecular weight, the number of atoms, the number of hydrogen donor atoms, the number of hydrogen acceptor atoms, and the polar surface area, of small molecules (http://www.molinspiration.com/cgi-bin/properties).

Active site prediction and docking analysis. Active site prediction is a state-of-the-art approach which helps in the determination of the sites/pockets/clefts in the protein structures, where any ligand or any small molecule can bind to it. Once the binding cleft or pocket is known, docking determines their affinity and binding stability and root mean square deviation (RMSD) value, etc. Active Site prediction tools, such as TM Site (12), is a tool used for the identification of the ligand binding template sequences from the BioLip protein functional database (13) by utilizing profile sequence differentiation and by attaching sub-structures (12); COACH (14) provides reliable results which can be used for docking analyses or any other research; COFACTOR (14) begins from the tertiary structure of the protein and threads the protein query through the BioLip functional database using global and local matches to determine functional and active sites within the query protein (14); these were employed for the purposes of this study. An online docking server, Docking Server (15), which is based on AutoDock, was employed for docking purposes thereafter. This online server has an inbuilt parameter which allows the user to select the site of docking in the target protein

Interaction analysis. After docking, many interactions are formed between a protein and its ligand, which are essential for the stability of the complex structure. The CCP4 tool (16) can be downloaded from the CCP4 suite from the main webpage (http://www.ccp4.ac.uk/docs.php. Once the tool is downloaded, the user finds that the tool has a simple GUI with a number of options, such as structural analysis, which again contains many options for studying the protein structures in detail (16). Protein Interaction Ligand Profiler (PLIP) (17), unlike other interactive tools, does not require any structural preparations and returns a list of interactions, mostly 7 types, such as hydrogen bonding, $\pi$-stacking, hydrophobic bonds, $\pi$-interactions and polar bonds.

\section{Results}

Pharmacokinetic, pharmacodynamic properties and bioactivity investigation. As a primary step towards confirming whether tanshinone compounds have the potential to be 'drug-like', i.e., it has properties such as solubility in water and other solvents, potency at the biological targets, ligand efficiency, and so on. It was exposed to Molinspiration prior to docking and interaction studies. The pharmacokinetic and physiochemical results are presented in Table VI. Pre-ADME evaluation showcases that tanshinone compounds are very much 'drug-like' as it does not violate any thumb rule, such as Lipinski's, Ghose's, Veber's and Eden's rule. Dihydrotanshinone I and cryptotanshinone have quite similar molecular weights (278.3 and $296.3 \mathrm{kDa}$, respectively), and their topological surface area was also equivalent viz., 43.38. Cryptotanshinone had an edge when compared to dihydrotanshinone I, as it was a better ion channel modulator. The plasma membrane has a bi-lipid layer, and only substances which are semi-permeable or completely permeable can pass through it. Cryptotanshinone being a good ion channel modulator, can gain access easily through the plasma membrane and can reach the other parts of the body easily via the blood.

As regards their bioactivity, it can be discerned that both exhibit a good overall bioactivity; however, cryptotanshinone again surpasses dihydrotanshinone. Bioactivity heavily depends on whether the ligand of interest is a G-protein coupled receptor (GPCR) ligand or not. GPCRs are mandatory as to when an external signaling molecule (ligand) binds to a GPCR; it leads a conformational alteration in the GPCR triggering an affiliation between the GPCR and a nearby $\mathrm{G}$ protein. This interaction is mandatory for the protein to become activated, in turn leading to secondary messaging in the body (16). Cryptotanshinone is a better GPCR ligand (0.24) when compared to dihydrotanshinone I (0.03).

AutoDock is a based online web-server; Docking Server was employed for docking. The results were obtained from this analysis may prove helpful in the opening of a new research 


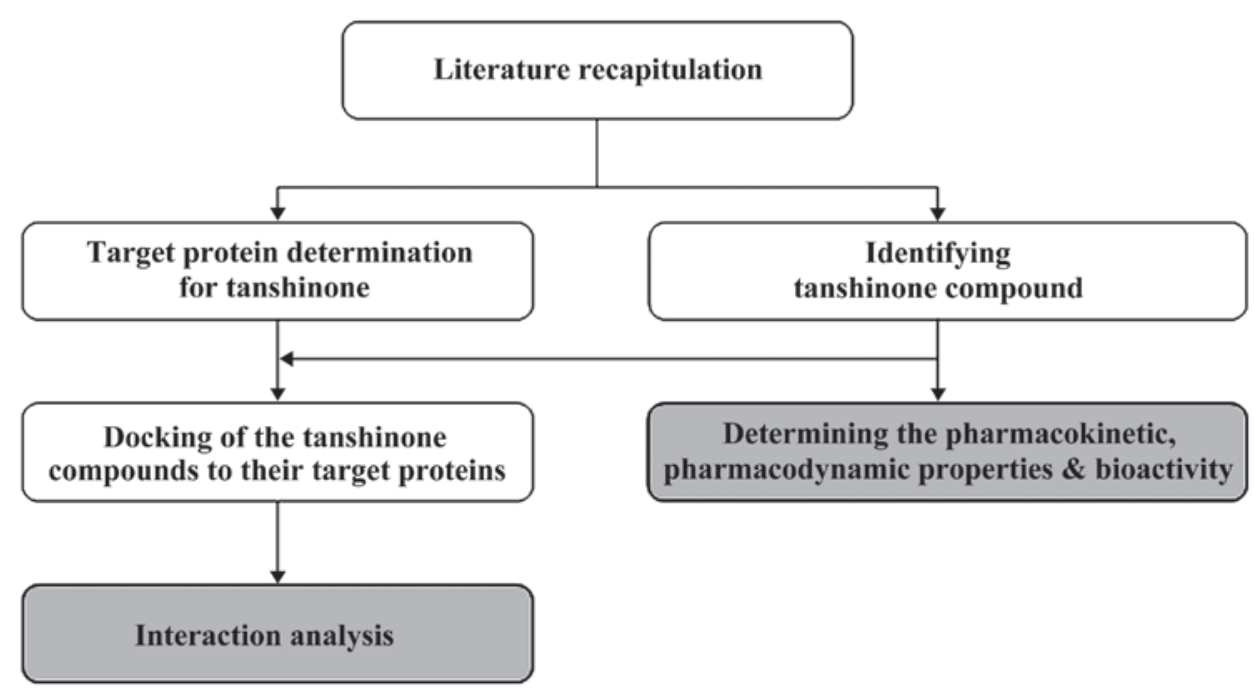

Figure 1. Flowchart of the basic operation mode adopted in this study.

Table I. List of target proteins for tanshinone IIA.

\begin{tabular}{|c|c|c|}
\hline ChEMBL ID & Description & UniProt ID \\
\hline ChEMBL4832 & Transcription initiation factor & P52655 \\
\hline ChEMBL3474 & Phospholipase A2 grp IIA & P14555 \\
\hline ChEMBL5841 & Low affinity immunoglobulin gamma-Fc region receptor IIA & P12318 \\
\hline ChEMBL2189151 & Myosin-9 & P35579 \\
\hline ChEMBL5616 & Activin receptor type $2 \mathrm{~A}$ & P27037 \\
\hline ChEMBL3797012 & Tubulin $\beta$-2A chain & Q13885 \\
\hline ChEMBL4444 & Vitamin K-dependent protein-C & P04070 \\
\hline ChEMBL1905 & Fibronectin receptor $\beta$ & P05556 \\
\hline ChEMBL3885597 & ITGB1-ITGA9 complex & PO5556, Q13797 \\
\hline ChEMBL3430895 & Integrin $\alpha-10 /$ itegrin beta- 1 & P05556, O75578 \\
\hline ChEMBL3137278 & Integrin $\alpha-1 / \beta-1$ complex & P05556, P56199 \\
\hline ChEMBL1907599 & Integrin $\alpha-4 / \beta-1$ & P05556, P13612 \\
\hline ChEMBL2111407 & Integrin $\alpha-V / \beta-1$ & P05556, P06756 \\
\hline ChEMBL3885596 & ITGA-6-ITGB1 complex & P05556, P23229 \\
\hline ChEMBL2095226 & Integrin $\alpha-5 / \beta-1$ & P05556, P08648 \\
\hline
\end{tabular}

Table II. List of target proteins for tanshinone IIA sulfone.

\begin{tabular}{llc}
\hline ChEMBL ID & & Description \\
\hline ChEMBL2265 & Acyl coenzyme A: cholesterol acyltransferase & UniProt ID \\
ChEMBL3180 & Carboxylesterase 2 & O00748 \\
ChEMBL1293237 & Bloom syndrome protein & P54132 \\
ChEMBL220 & Acetylcholinesterase & P22303 \\
ChEMBL5365 & DNA Pol kappa & Q9UBT6 \\
ChEMBL5990 & Breast cancer type I susceptibility protein & P38398 \\
ChEMBL1795087 & Ubiquitin carboxyl terminal hydrolase I & O94782 \\
ChEMBL2903 & Archidonate 15-lipoxygenase & P16050 \\
ChEMBL4159 & Endoplasmic reticulum-associated amyloid beta peptide binding protein & Q99714 \\
ChEMBL1293255 & 15-hydroxyprostaglandin dehydrogenase (NAD ${ }^{+}$ & P15428 \\
ChEMBL340 & Cytochrome P4503A4 & P08684 \\
ChEMBL3577 & Aldehyde dehydrogenase1A1 & P00352 \\
\hline
\end{tabular}


Table III. List of target proteins for dihydrotanshinone I.

\begin{tabular}{lll}
\hline ChEMBL ID & \multicolumn{1}{c}{ Description } & UniProt ID \\
\hline ChEMBL2265 & Acyl coenzyme A: cholesterol acyltransferase & P23141 \\
ChEMBL3180 & Carboxylesterase 2 & O00748 \\
ChEMBL3166 & Protein-tyrosine phosphatase 1C & P29350 \\
ChEMBL220 & Acetylcholinesterase & P22303 \\
ChEMBL5365 & DNA Pol kappa & Q9UBT6 \\
ChEMBL3864 & Protein-tyrosine phosphatase 2C & Q06124 \\
ChEMBL1907606 & Mitogen-activated protein kinase ERK1/ERK2 & P27361, P28482 \\
ChEMBL2094115 & MAP Kinase 38 (Family protein) & Q16539, P53778 \\
ChEMBL1075138 & Tyrosyl DNA phosphodiesterase & Q9NUW8
\end{tabular}

Table IV. List of target proteins for cryptotanshinone.

\begin{tabular}{llc}
\hline ChEMBL ID & \multicolumn{1}{c}{ Description } & UniProt ID \\
\hline ChEMBL2265 & Acyl coenzyme A: cholesterol acyltransferase & P23141 \\
ChEMBL3180 & Carboxylesterase 2 & O00748 \\
ChEMBL3166 & Protein-tyrosine phosphatase 1C & P29350 \\
ChEMBL220 & Acetylcholinesterase & P22303 \\
ChEMBL5365 & DNA Pol kappa & Q9UBT6 \\
ChEMBL3864 & Protein-tyrosine phosphatase 2C & Q06124 \\
ChEMBL2916 & Telomerase reverse transcriptase & O14746 \\
ChEMBL5027 & Flap endonuclease 1 & P39748 \\
ChEMBL6117 & Tyrosine-protein phosphatase non-receptor type 9 & P43378 \\
ChEMBL3521 & Receptor type tyrosine-protein phosphatase F (LAR) & P10586 \\
ChEMBL3807 & T-cell protein tyrosine phosphatase & P17706 \\
ChEMBL3243 & Leukocyte common antigen & P08575 \\
ChEMBL1293236 & ATP-dependent DNA helicase Q1 & P46063 \\
ChEMBL335 & Protein tyrosine phosphatase 1B & P18031 \\
ChEMBL2146312 & Werner syndrome ATP-dependent helicase & Q14191 \\
\hline
\end{tabular}

Table V. List of common target proteins for dihydrotanshinone I and cryptotanshinone.

\begin{tabular}{llc}
\hline ChEMBL ID & \multicolumn{1}{c}{ Description } & UniProt ID \\
\hline ChEMBL2265 & $\begin{array}{l}\text { Acyl coenzyme A: } \\
\text { cholesterol acyltransferase }\end{array}$ & P23141 \\
ChEMBL3180 & Carboxylesterase 2 & O00748 \\
ChEMBL220 & Acetylcholinesterase & P22303 \\
ChEMBL5365 & DNA Pol kappa & Q9UBT6 \\
\hline
\end{tabular}

avenues. It is known that the ligands, dihydrotanshinone I and cryptotanshinone hold a surface binding to 4 target proteins. Figs. 2 and 3 showcase the surface binding of dihydrotanshinone I and cryptotanshinone with P23141, O00748, P22303 and Q9UBT6, which refers to the planar surface of the docking site of the target proteins. Furthermore, it hints to a rigid docking, which is surely a rare case. Interaction analysis discerned that the ligands carry out mainly 4 types of interactions with the receptor proteins namely, hydrogen bonds, hydrophobic interac- tions, polar bonds and $\pi-\pi$ interactions. The complete docking affinities and energy estimates re provided in Table VII, while interaction details are presented in Table VIII.

Many other interactions were formed apart from the ones mentioned in Table VIII. Figs. 4 and 5 encapsulate the interaction plots for all the 4 target proteins with cryptotanshinone and dihydrotanshinone I, respectively. Exploration and exploitation are the two key factors in data analysis. HBD plots are simply interactive plots which show interactions of amino acid residues with the ligand. They help to understand the interaction quality between the receptors and ligands, which in turn helps to learn more about the flexible nature of the protein domains. The interactive plots shown in Fig. 4 clearly indicate that cryptotanshinone has a better affinity and binding to its target receptors. The interactions also suggest that the docking between cryptotanshinone and O00748, P22303, P23141 and Q9UBT6 is a flexible docking.

\section{Discussion}

The in silico approach has aimed to place some credit on the anti-inflammatory-based herbal compound, tanshinone. 
Table VI. Bioactivity, pharmacokinetic and pharmacodynamic properties of dihydrotanshinone I and cryptotanshinone.

A, Bioactivity

\begin{tabular}{|c|c|c|c|c|c|c|}
\hline Compound & $\begin{array}{l}\text { GPCR } \\
\text { ligand }\end{array}$ & $\begin{array}{l}\text { Ion xhannel } \\
\text { modulator }\end{array}$ & $\begin{array}{l}\text { Kinase } \\
\text { inhibitor }\end{array}$ & $\begin{array}{c}\text { Nuclear receptor } \\
\text { ligand }\end{array}$ & $\begin{array}{l}\text { Protease } \\
\text { inhibitor }\end{array}$ & $\begin{array}{l}\text { Enzyme } \\
\text { inhibitor }\end{array}$ \\
\hline Dihydrotanshinone I & 0.03 & 0.00 & 0.04 & 0.09 & -0.15 & 0.28 \\
\hline Cryptotanshinone & 0.24 & 0.36 & -0.07 & 0.31 & -0.14 & 0.46 \\
\hline
\end{tabular}

B, Pharmacokinetic and pharmacodynamic properties

\begin{tabular}{lccccccr}
\hline Compound & miLogP & TPSA & natoms & MW & nON & nOHNH & nViolations \\
\hline Dihydrotanshinone I & 3.51 & 43.38 & 21 & 278.3 & 3 & 0 & 0 \\
Cryptotanshinone & 3.83 & 43.38 & 22 & 296.3 & 3 & 0 & 247.4 \\
\hline
\end{tabular}

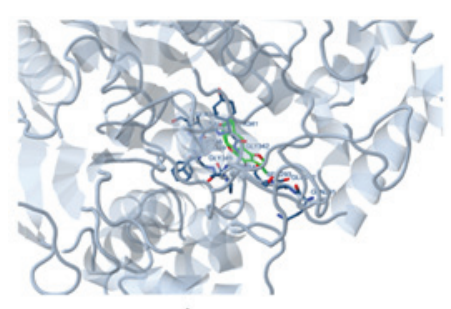

Cryptotanshinone to $\mathrm{P} 22303$

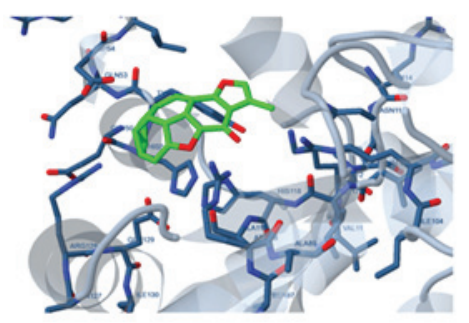

Cryptotanshinone to 000748

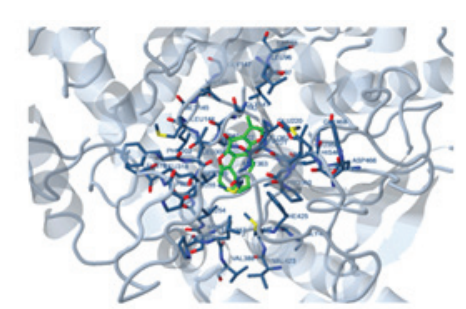

Cryptotanshinone to $\mathrm{P} 23141$

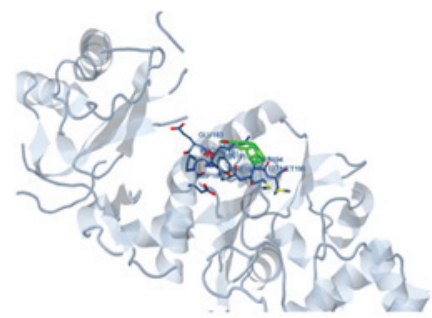

Cryptotanshinone to Q9UBT6

Figure 2. Surface docking shown by cryptotanshinone to its target proteins.

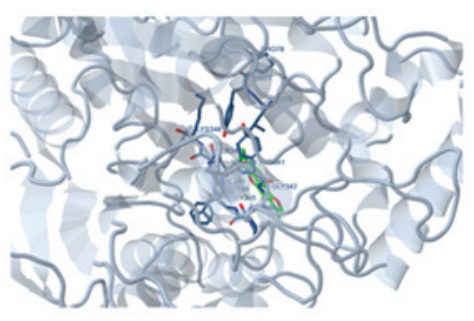

Dihydrotanshinone I to P22303

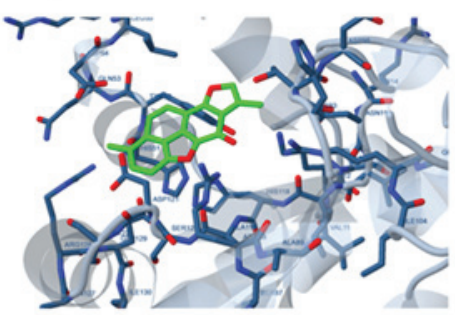

Dihydrotanshinone I to 000748

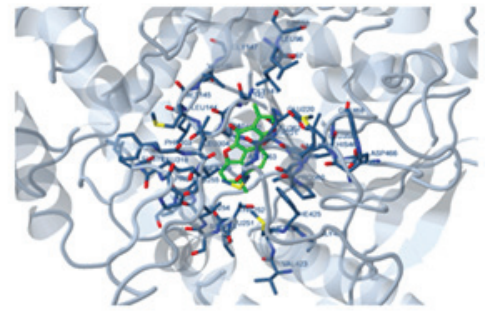

Dihydrotanshinone I to P23141

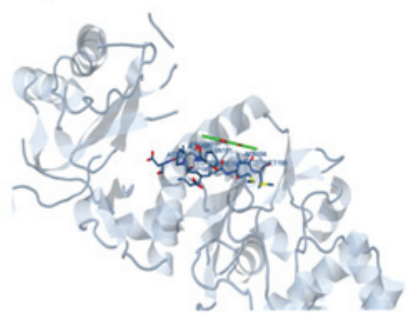

Dihydrotanshinone I to Q9UBT6

Figure 3. Rigid and a surface docking displayed by dihydrotanshione I to its target proteins. 


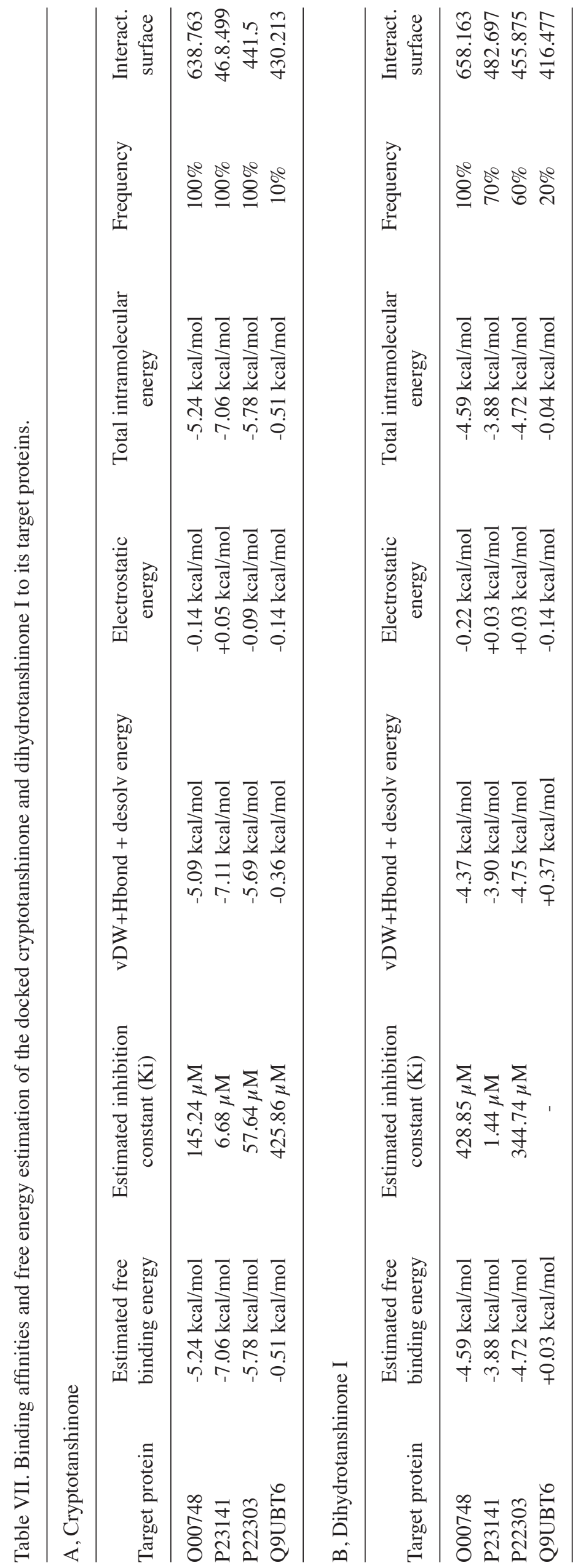


Table VIII. Interactions formed by dihydrotanshinone I and cryptotanshinone with the receptor targets, O00748, P23141, P22303 and Q9UBT6.

A, Dihydrotanshinone I

\begin{tabular}{|c|c|c|c|}
\hline Hydrogen bonds & Hydrophobic bonds & $\pi-\pi$ Interactions & Polar bonds \\
\hline \multicolumn{4}{|l|}{ O00748 } \\
\hline O2()-ARG105 & C1()-LEU55 & $\begin{array}{l}\text { C1()-TYR52; C10()-TYR52; } \\
\text { C14()-TYR52; C4()-HIS118; } \\
\text { C6()-HIS118, C12()-HIS118 }\end{array}$ & $\begin{array}{l}\text { O2()-ARG88; } \\
\text { O3()-ARG88; } \\
\text { O3()-HIS11 }\end{array}$ \\
\hline \multicolumn{4}{|l|}{ P23141 } \\
\hline & $\begin{array}{l}\text { C17()-LEU97; C15()-LEU97; C16()-LEU97; } \\
\text { C17()-VAL146; C7()-VAL254; C6()-VAL254; } \\
\text { C8()-VAL254; C18()-VAL254; C6()-LEU255; } \\
\text { C7()_LEU255; C5()-LEU318; C6()-LEU318; } \\
\text { C15()-358; C1()-ILE359; C2()-ILE359; } \\
\text { C13()-LEU362; C15()-ILE362, C16()-ILE362; } \\
\text { C17()-ILE362; C8()-MET424; C18()-MET424; } \\
\text { C18()-PHE425 }\end{array}$ & $\begin{array}{l}\text { C2()-PHE425; C1()-HIS467; } \\
\text { C2()-HIS467 }\end{array}$ & O1()-HIS467 \\
\hline \multicolumn{4}{|l|}{ P22303 } \\
\hline & $\begin{array}{l}\text { C18()-TYR77; C13()-PRO344; C16()-PRO344; } \\
\text { C17()-PRO344 }\end{array}$ & & \\
\hline \multicolumn{4}{|l|}{ Q9UBT6 } \\
\hline O1()-ALA194 & $\begin{array}{l}\text { C18()-ALA186; C18()-PHE192; C1()-ALA194; } \\
\text { C10()-ALA194; C14()-ALA194 }\end{array}$ & $\begin{array}{l}\text { C3()-PHE192; C8()-PHE192; } \\
\text { C1()-PHE192; C2()-PHE192; } \\
\text { C7()-PHE192 }\end{array}$ & \\
\hline
\end{tabular}

B, Cryptotanshinone

\begin{tabular}{|c|c|c|c|}
\hline Hydrogen bonds & Hydrophobic bonds & $\pi-\pi$ Interactions & Polar bonds \\
\hline \multicolumn{4}{|l|}{ O00748 } \\
\hline & $\begin{array}{l}\text { C19()-LEU97; C17()-LEU97; C18()-LEU97; } \\
\text { C19()-VAL146; C7()-VAL254; C8()-VAL254; } \\
\text { C13()-LEU304; C14()-LEU304; C5()-LEU318; } \\
\text { C6()-LEU318; C17()-LEU358; C1()-ILE359, } \\
\text { C9()-ILE359, C10()-ILE359; C17()-LEU362; } \\
\text { C18()-LEU362; C19()-LEU362; C7()-LEU387; } \\
\text { C9()-387; C7()-MET424; C8()-MET424; } \\
\text { C9()-MET424; C10()-MET424; C1()-HIS467 }\end{array}$ & C10()-PHE425 & O1()-HIS467 \\
\hline \multicolumn{4}{|l|}{ P23141 } \\
\hline & $\begin{array}{l}\text { C19()-LEU97; C17()-LEU97; C18()-LEU97; } \\
\text { C19()-VAL146; C7()-VAL254; C8()-VAL254; } \\
\text { C13()-LEU304; C14()-LEU304; C5()-LEU318; } \\
\text { C6()-LEU318; C17()-LEU358; C1()-ILE359, } \\
\text { C9()-ILE359, C10()-ILE359; C17()-LEU362; } \\
\text { C18()-LEU362; C19()-LEU362; C7()-LEU387; } \\
\text { C9()-387; C7()-MET424; C8()-MET424; } \\
\text { C9()-MET424; C10()-MET424; C1()-HIS467 }\end{array}$ & C10()-PHE425 & O1()-HIS467 \\
\hline \multicolumn{4}{|l|}{ P22303 } \\
\hline \multicolumn{4}{|l|}{ Q9UBT6 } \\
\hline O2()-LYS182 & $\begin{array}{l}\text { C19()-PHE192; C7()-MET193; C9()-MET193; } \\
\text { C6()-MET193; C13()-ALA194 }\end{array}$ & $\begin{array}{l}\text { C17()-PHE192; C18()-PHE192; } \\
\text { C15()-PHE192 }\end{array}$ & \\
\hline
\end{tabular}




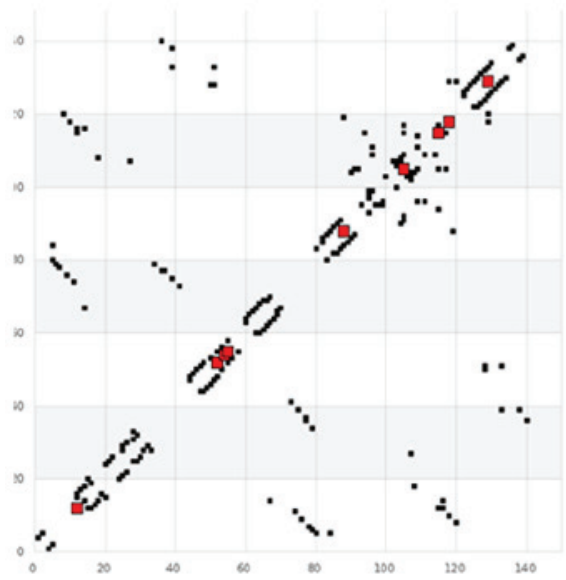

Cryptotanshinone to P23141

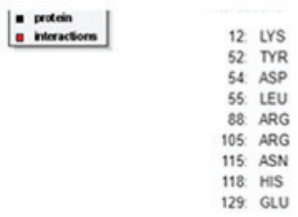

Cryptotanshinone to $\mathrm{O} 00748$
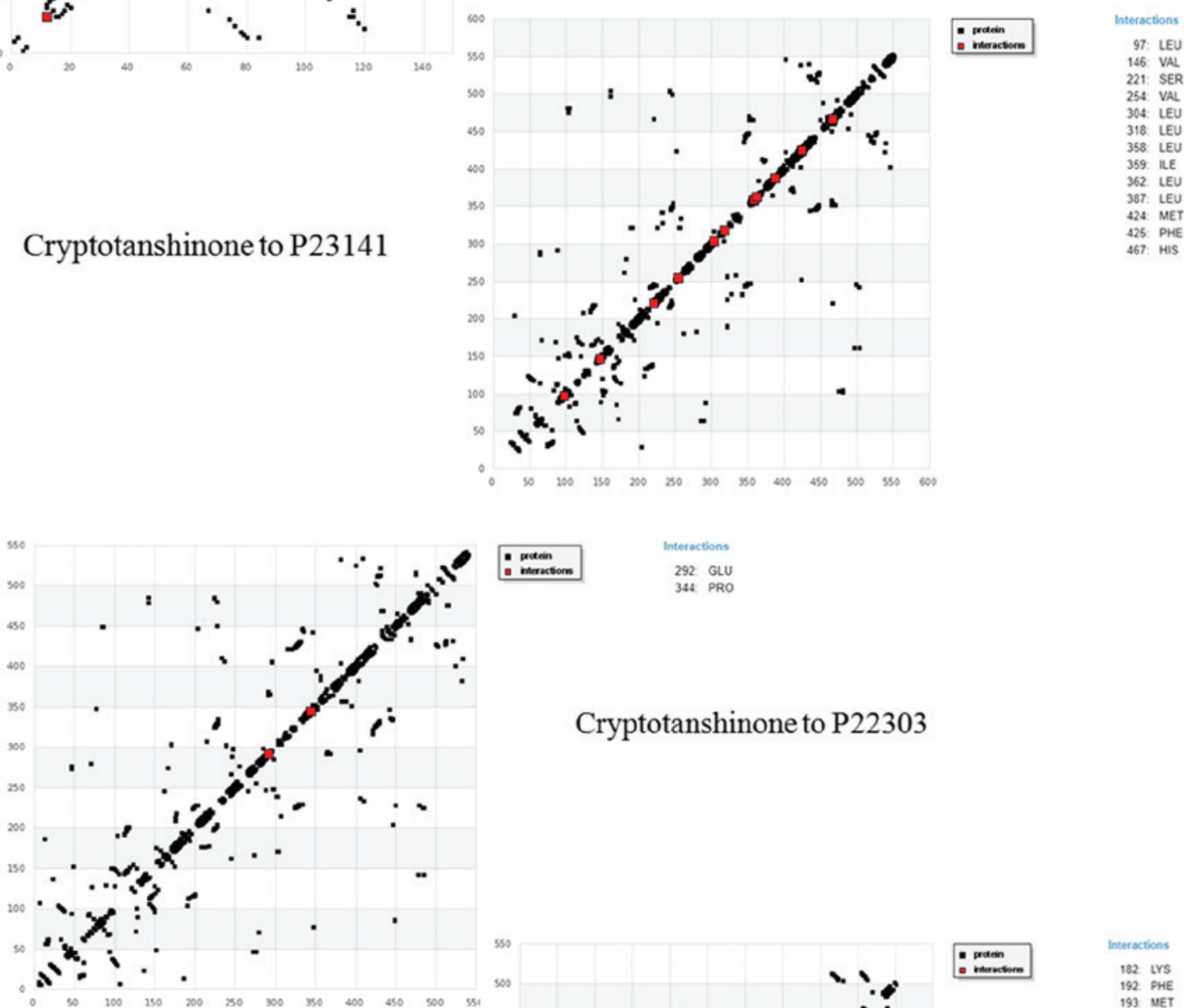

Cryptotanshinone to P22303

Cryptotanshinone to Q9UBT6
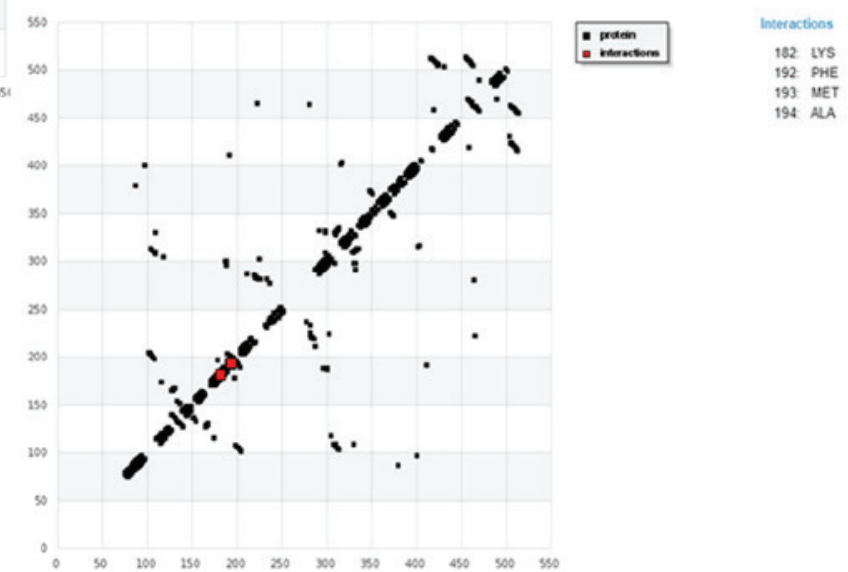

Figure 4. HBD interactive plot of cryptotanshinone with target receptors.

Through various bioinformatics techniques, we can identify and modify the drugs that are useful for the treatment of inflammation, and this may lead to novel insight being obtained into new aspects of anti-inflammatory disorders, which are less known to society, indicating vindictively the fact that our how society carries a lackadaisical approach towards such issues. It is important to be up well informed and to carry out detailed investigations of the proteins involved in anti-inflammation 

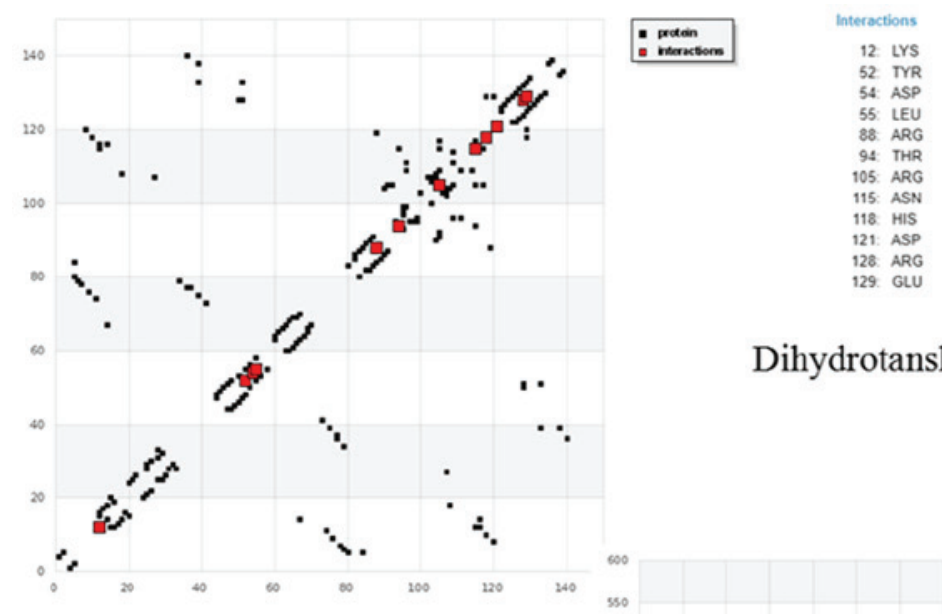

\section{Dihydrotanshinone I to O00748}

Dihydrotanshinone I to P23141

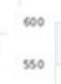
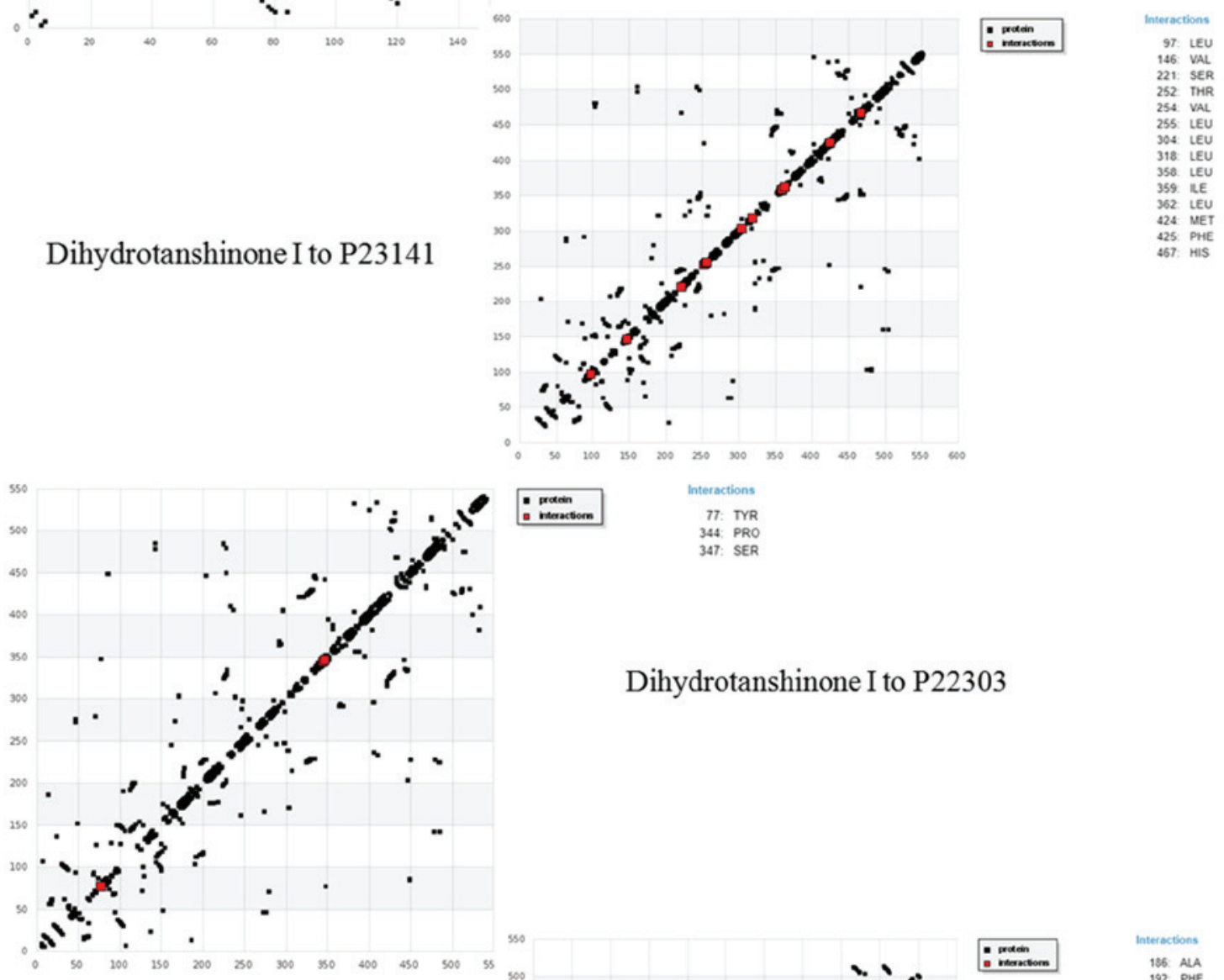

Dihydrotanshinone I to P22303

Dihydrotanshinone I to Q9UBT6
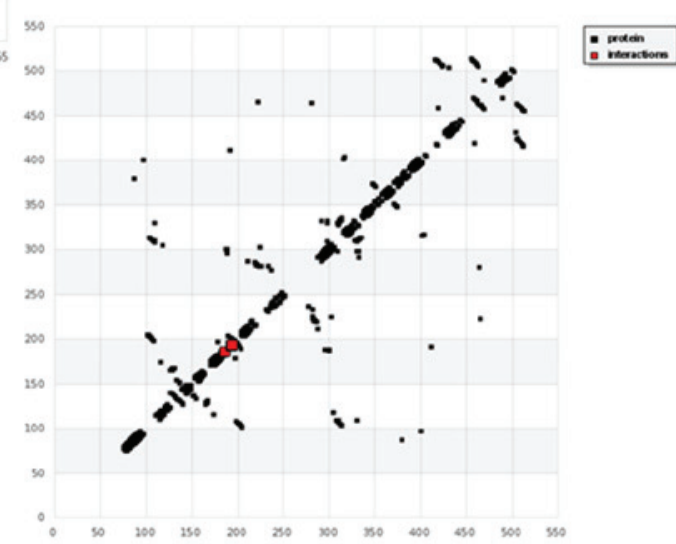

186. ALA
192 PPE
194 ALA

Figure 5. HBD interactive plot of dihydrotanshinone I with target receptors.

and of the traditional herbal medications which are being used to treat this disorder. A great amount of knowledge has been gained which needs to be synergistically collated and discussed. A gateway of research for elucidating inflammation and its treatment using herbal medicines was the main lead in this study. More than 15 protein targets were retrieved out of which only 4 target receptors were selected and examined. Along with this, lipid-soluble compounds, which were specific 
to the tanshinone class, which are being used in the treatment of inflammation were also highlighted, out of which, 2 compounds, dihydrotanshinone I and cryptotanshinone were selected with an aim of providing a new research avenue for the elucidation of the detailed mechanisms responsible for the pathogenesis of inflammatory diseases. These are multi-faceted herbal compounds whose benefits can be extracted and can be utilized effectively for human well-being and healthcare. From the treatment of breast cancer to inflammatory diseases, a herbal medication can prove to be effective. It is surely their agrarian nature which makes these medications a 'blessing in disguise'. In this study, an imperforate strategy was laid down in order to recognize the abilities of dihydrotanshinone I and crytptotanshinone with their pharmacokinetic, pharmacodynamic and physiocochemical properties, and ultimately to expose the ligands and the receptors proteins to docking analysis and to determine the binding affinity. Moreover, interactions analyses were accomplished successfully to identify the interactions formed between both dihdrotanshinone I and cryptotanshinone, and the 4 target proteins.

The bioactivity properties and various other pharmacokinetic and pharmacodynamic analyses discerned that crytptotanshinone was more effective than dihydrotanshinone and was more 'drug-like' than its counterpart. It has a better solubility, permeability and holds potential for use as an anti-inflammatory agent.

Docking analysis and interaction analyses were performed and revealed that there is an affinity between cryptotanshinone and O00748, P23141 and Q9UBT6, and interactions such as hydrogen bonds, $\pi-\pi$ bonds, hydrophobic bonds are formed between the ligand and the receptor proteins. Hydrogen bonding between the ligand and the proteins indicate that the complexes have a good stability.

\section{Acknowledgements}

Not applicable.

\section{Funding}

This study was supported by the Medical cross project of Shanghai Jiao Tong University (no. YG2016QN74).

\section{Availability of data and materials}

The datasets used and/or analyzed during the current study are available from the corresponding author on reasonable request.

\section{Authors' contributions}

ZR, LN and LH conceived and designed the study. ZR, LN, $\mathrm{LH}, \mathrm{RR}, \mathrm{ZX}, \mathrm{WD}$ and $\mathrm{LJ}$ were responsible for the collection and assembly of data, data analysis and interpretation. ZR, LN and LH were involved in the writing of the manuscript. All authors have read and approved the final manuscript.

\section{Ethics approval and consent to participate}

Not applicable.

\section{Patient consent for publication}

Not applicable

\section{Competing interests}

The authors declare that they have no competing interests.

\section{References}

1. Zhang Y, Jiang P, Ye M, Kim SH, Jiang C and Lü J: Tanshinones: Sources, pharmacokinetics and anti-cancer activities. Int J Mol Sci 13: 13621-13666, 2012.

2. Ji XY, Kwong-Huat Tan B, Huang SH, Whiteman M, Zhu YC, Linz W, Duan W and Zhu YZ: Effects of Salvia miltiorrhiza after acute myocardial infarction in rats. In: Novel Compounds from Natural Products in the New Millennium. World Scientific Publishing Co. Pte Ltd., Singapore, pp183-195, 2004.

3. Li D, Wang J, Sun D, Gong X, Jiang H, Shu J, Wang Z, Long Z, Chen Y, Zhang Z, et al: Tanshinone IIA sulfonate protects against cigarette smoke-induced COPD and down-regulation of CFTR in mice. Sci Rep 8: 376, 2018.

4. Guan R, Wang J, Li Z, Ding M, Li D, Xu G, Wang T, Chen Y, Yang Q, Long Z, et al: Sodium Tanshinone IIA Sulfonate Decreases Cigarette Smoke-Induced Inflammation and Oxidative Stress via Blocking the Activation of MAPK/HIF-1 $\alpha$ Signaling Pathway. Front Pharmacol 9: 263, 2018.

5. Gao H, Sun W, Zhao J, Wu X, Lu JJ, Chen X, Xu QM, Khan IA and Yang S: Tanshinones and diethyl blechnics with anti-inflammatory and anti-cancer activities from Salvia miltiorrhiza Bunge (Danshen). Sci Rep 6: 33720, 2016.

6. Staniforth V, Wang SY, Shyur LF and Yang NS: Shikonins, phytocompounds from Lithospermum erythrorhizon, inhibit the transcriptional activation of human tumor necrosis factor alpha promoter in vivo. J Biol Chem 279: 5877-5885, 2004.

7. Chen W, Lu Y, Chen G and Huang S: Molecular evidence of cryptotanshinone for treatment and prevention of human cancer. Anticancer Agents Med Chem 13: 979-987, 2013.

8. Kim S, Chen J, Cheng T, Gindulyte A, He J, He S, Li Q, Shoemaker BA, Thiessen PA, Yu B, et al: PubChem 2019 update: Improved access to chemical data. Nucleic Acids Res 47 (D1): D1102-D1109, 2019.

9. Gaulton A, Bellis LJ, Bento AP, Chambers J, Davies M, Hersey A, Light Y, McGlinchey S, Michalovich D, Al-Lazikani B, et al: ChEMBL: A large-scale bioactivity database for drug discovery. Nucleic Acids Res 40 (D1): D1100-D1107, 2012.

10. Law V, Knox C, Djoumbou Y, Jewison T, Guo AC, Liu Y, Maciejewski A, Arndt D, Wilson M, Neveu V, et al: DrugBank 4.0: Shedding new light on drug metabolism. Nucleic Acids Res 42 (D1): D1091-D1097, 2014

11. Wishart DS, Knox C, Guo AC, Cheng D, Shrivastava S, Tzur D, Gautam B and Hassanali M: DrugBank: A knowledgebase for drugs, drug actions and drug targets. Nucleic Acids Res 36 (Suppl 1): D901-D906, 2008.

12. Xu J and Zhang Y: How significant is a protein structure similarity with TM-score $=0.5$ ? Bioinformatics $26: 889-895,2010$

13. Roy A, Yang J and Zhang Y: COFACTOR: An accurate comparative algorithm for structure-based protein function annotation. Nucleic Acids Res 40 (W1): W471-477, 2012.

14. Yang J, Roy A and Zhang Y: BioLiP: A semi-manually curated database for biologically relevant ligand-protein interactions. Nucleic Acids Res 41 (D1): D1096-D1103, 2013.

15. Bikadi Z and Hazai E: Application of the PM6 semi-empirical method to modeling proteins enhances docking accuracy of AutoDock. J Cheminform 1: 15, 2009.

16. Winn MD, Ballard CC, Cowtan KD, Dodson EJ, Emsley P, Evans PR, Keegan RM, Krissinel EB, Leslie AG, McCoy A, et al: Overview of the CCP4 suite and current developments. Acta Crystallogr D Biol Crystallogr 67: 235-242, 2011.

17. Salentin S, Schreiber S, Haupt VJ, Adasme MF and Schroeder M: PLIP: Fully automated protein-ligand interaction profiler. Nucleic Acids Res 43 (W1): W443-447, 2015. International (CC BY-NC-ND 4.0) License. 